\title{
Method for temporal keyhole lobectomies in resection of low- and high-grade gliomas
}

\author{
Andrew K. Conner, MD, ${ }^{1}$ Joshua D. Burks, BA, ${ }^{1}$ Cordell M. Baker, BA, ${ }^{1}$ \\ Adam D. Smitherman, MD, ${ }^{1}$ Dillon P. Pryor, BA, ${ }^{1}$ Chad A. Glenn, MD, ${ }^{1}$ Robert G. Briggs, BS, ${ }^{1}$ \\ Phillip A. Bonney, MD, ${ }^{2}$ and Michael E. Sughrue, MD' \\ 12Department of Neurosurgery, University of Oklahoma Health Sciences Center, Oklahoma City, Oklahoma; and 2Department of \\ Neurological Surgery, University of Southern California, Los Angeles, California
}

\begin{abstract}
OBJECTIVE The purpose of this study was to describe a method of resecting temporal gliomas through a keyhole lobectomy and to share the results of using this technique.

METHODS The authors performed a retrospective review of data obtained in all patients in whom the senior author performed resection of temporal gliomas between 2012 and 2015. The authors describe their technique for resecting dominant and nondominant gliomas, using both awake and asleep keyhole craniotomy techniques.

RESULTS Fifty-two patients were included in the study. Twenty-six patients (50\%) had not received prior surgery. Seventeen patients (33\%) were diagnosed with WHO Grade II/III tumors, and 35 patients $(67 \%)$ were diagnosed with a glioblastoma. Thirty tumors were left sided (58\%). Thirty procedures (58\%) were performed while the patient was awake. The median extent of resection was $95 \%$, and at least $90 \%$ of the tumor was resected in 35 cases (67\%). Five of 49 patients (10\%) with clinical follow-up experienced permanent deficits, including 3 patients $(6 \%)$ with hydrocephalus requiring placement of a ventriculoperitoneal shunt and 2 patients (4\%) with weakness. Three patients experienced early postoperative anomia, but no patients had a new speech deficit at clinical follow-up.
\end{abstract}

CONCLUSIONS The authors provide their experience using a keyhole lobectomy for resecting temporal gliomas. Their data demonstrate the feasibility of using less invasive techniques to safely and aggressively treat these tumors.

https://thejns.org/doi/abs/10.3171/2016.12.JNS162168

KEY WORDS keyhole; glioma; craniotomy; temporal lobe; resection; minimally invasive; oncology

$\mathrm{T}$ TERE is increasing interest in developing surgical methods that are less traumatic, with the hope that these procedures will hasten patient recovery. Keyhole craniotomy is a surgical technique that was developed in an attempt to minimize the incision and craniotomy needed to access intracranial pathologies. The keyhole approach uses a craniotomy that opens interiorly as a "reversed funnel-shaped surgical corridor." 25 This is in contrast to traditional craniotomies, which require a larger opening that funnels to the point of interest. Despite the less invasive nature of this technique, many surgeons believe that a limited-size approach carries higher risks due to inadequate visualization of critical structures and restricted ability to control intraoperative hemorrhage. ${ }^{20}$

Surgical visualization of relevant anatomy is critical for safe and effective neurosurgery, although what constitutes adequate visualization has not been effectively delineated. We argue that the ideal approach is the least destructive

ABBREVIATIONS DTI = diffusion tensor imaging; EOR = extent of resection; IFOF = inferior frontooccipital fasciculus; POD = postoperative day; $S L F=$ superior longitudinal fasciculus; $S T G=$ superior temporal gyrus.

SUBMITTED August 21, 2016. ACCEPTED December 21, 2016.

INCLUDE WHEN CITING Published online July 7, 2017; DOI: 10.3171/2016.12.JNS162168. 
method that can safely and effectively meet the demands of the case being performed.

We have developed a method for temporal lobectomies using a keyhole craniotomy in patients with low- and highgrade gliomas. This article provides data on the technical aspects of temporal keyhole craniotomies and details the outcomes of patients who have undergone an operation that involves using this less invasive technique. In this study, we showed that the keyhole method is a reasonable approach to glioma resection, with results comparable to those of traditional surgical methods.

\section{Methods \\ Patient Selection}

We performed a retrospective review of data in all patients who underwent a temporal keyhole craniotomy; the senior author (M.E.S.) performed these operations between 2012 and 2015 at the University of Oklahoma Health Sciences Center. We included all patients with a histopathological diagnosis of diffuse low-grade glioma (WHO Grade II), anaplastic oligodendroglioma/astrocytoma (WHO Grade III), or glioblastoma, who underwent resection. Biopsy-only patients and those with insular tumors were not included in the study. Clinical records, hospital charts, and imaging studies were reviewed through the last available follow-up. Patients who were not seen at least 3 months postoperatively were noted as lost to followup. Medical history, operative notes, and hospital course were also reviewed. This study was performed with the approval of our institutional review board.

\section{Preoperative Assessment and Mapping}

MRI with and without Gd contrast was performed preoperatively in all patients. Using diffusion tensor imaging (DTI) tractography, the corticospinal tract, superior longitudinal fasciculus (SLF), arcuate fasciculus, inferior frontooccipital fasciculus (IFOF), and optic pathways were included in image guidance for operative planning and resection, as described elsewhere. ${ }^{5}$ Patients underwent preoperative clinical evaluation by physical and speech therapists, including spatiotemporal, attention, speech, and motor testing. The decision to perform awake surgery, as described by others, ${ }^{14}$ was made based on tumor proximity to eloquent structures. In the temporal lobe, attention was given to tumors in speech areas, specifically involving the posterior SLF, temporal cortical terminus of the SLF, superior temporal gyrus, subinsular IFOF, angular gyrus, and supramarginal gyrus. To ensure preservation of these eloquent structures, this study used intraoperative cortical and subcortical stimulation.

Our mapping paradigm primarily uses negative mapping, as described by others. ${ }^{32}$ During subcortical mapping, the patient was kept awake through the entirety of the cuts to the critical parts of the subcortical cortex. The key was to separate the temporal lobe from the speech networks and the IFOF/SLF/arcuate fasciculus until the ventricle was reached. Subcortical mapping and feedback determined the exact location of the cuts during dissection. During dissection, the patient was doing a double task that involved arm motor movement and continuous naming or reading, depending on the cortical mapping.
Prior to extending the cut in any particular direction, we performed stimulation of the subcortex with the stimulator. A negative site was confirmed by at least 3 separate stimulations at an average current of $4 \mathrm{~mA}$. We chose 4 $\mathrm{mA}$ based on our experience that this current is sensitive in identifying the eloquent cortex and introduces minimal risk of seizure. If a site at the planned disconnection was confirmed as negative, then it was included in the resection. Once the cuts connected the ventricle to the skull base, the patient was rendered asleep and the temporal lobectomy was performed. Again, preoperative planning using tractography is crucial to our method. If a patient was unable to undergo awake mapping, then diffusion tractography was used to guide the resection without the confirmatory aid of negative mapping.

There is evidence to support both negative and positive mapping in the setting of glioma resection. ${ }^{31,32}$ However, it is beyond the scope of this study to justify use of one technique over the other. Our findings are presented within the context of negative mapping, because this is the standard of care at our institution.

\section{Surgical Technique}

The premise of a keyhole temporal glioma lobectomy is that the majority of the risk in a large temporal lobe resection occurs with the posterior disconnection among the tumor, speech networks, and cortices. A subcortical disconnection is performed with an L-shaped dissection and is planned anterior to the SLF. Awake cortical mapping followed by subcortical mapping is performed. In the nondominant temporal lobe at the superior temporal gyrus (STG) and supramarginal gyrus, awake line-bisection and target cancelation tasks are done to negatively map for visuospatial function. ${ }^{6}$ As subcortical dissection is performed on either the dominant or nondominant temporal lobe, the patient is instructed to perform a double task (2 different modalities stressing 2 systems, e.g., naming and contralateral motor movement). ${ }^{19}$ This is done continuously until the disconnection to the temporal horn is completed. In our experience, having patients perform a double task increases the sensitivity in identifying eloquent regions, thereby demonstrating subtle deficits earlier than other methods. Dissection is continued below the subinsular region, avoiding the IFOF, which is known to reside there. ${ }^{23}$ The IFOF is identified with subcortical mapping using DTI integrated with image guidance and is confirmed with stimulation if needed.

The posterior disconnection is complete after 1) entering the ventricle, 2) continuing the cut to the front of the middle fossa, and 3) removal of the STG from anterior to posterior. We preserve the hippocampus as a temporary landmark to help define the superiorly located basal ganglia. It is removed at the completion of the lobectomy.

With those disconnections in mind, the keyhole craniotomy was planned over the posterior and superior aspect of the tumor temporal cortex boundary at the planned division site using image guidance, as shown in Fig. 1. We ensured that the superior edge of the craniotomy allowed exposure of the inferior-most aspect of the operculum. The inferior edge of the craniotomy allowed exposure to the center of the middle temporal gyrus in most cas- 

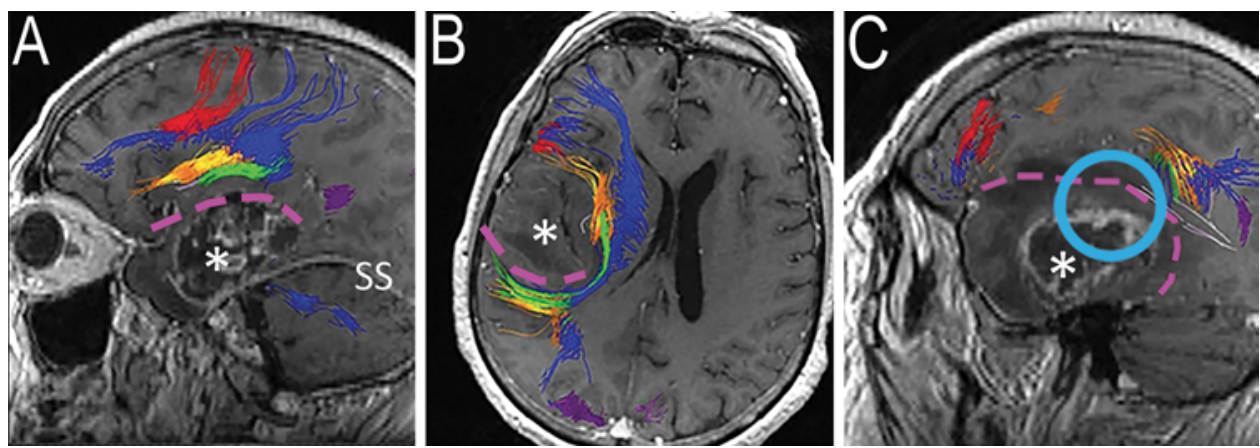

FIG. 1. Surgical planning. Using DTI preoperatively, the craniotomy was planned with respect to the white matter anatomy. A: Sagittal MR image showing the superior planned disconnection, represented by a pink dashed line; this was made below the sylvian fissure with avoidance of the inferior longitudinal fasciculus in green. B: Axial MR image showing the posterior planned disconnection, represented by a pink dashed line. This disconnection was planned anterior to the tracts lying posterior to the tumor and lateral to the tracts lying medial of the tumor. C: Using the trajectories of the planned surgical disconnections from $A$ and $B$ (represented by a pink dashed line), the craniotomy was positioned to allow access to the anterior temporal pole and inferior temporal boundary. The craniotomy site is illustrated with a light blue circle. White asterisk = tumor location. SS = straight sinus.

es. The inferior temporal cortex, anterior temporal lobe, and anterior superior temporal lobe (next to the sylvian fissure) were not entirely exposed but lay under the bone flap edge of the craniotomy. Angles close enough to the anterior superior STG or to the front of the sylvian fissure were considered when planning the anterior aspect of the craniotomy. This emphasizes the importance of preoperative planning with respect to the limits of the SLF. This is illustrated in Fig. 2. Once the craniotomy was planned, a linear incision of $5 \mathrm{~cm}$ was made in the coronal plane. The scalp was retracted with the aid of multiple scalp hooks attached to Penrose drains. A single small bur hole

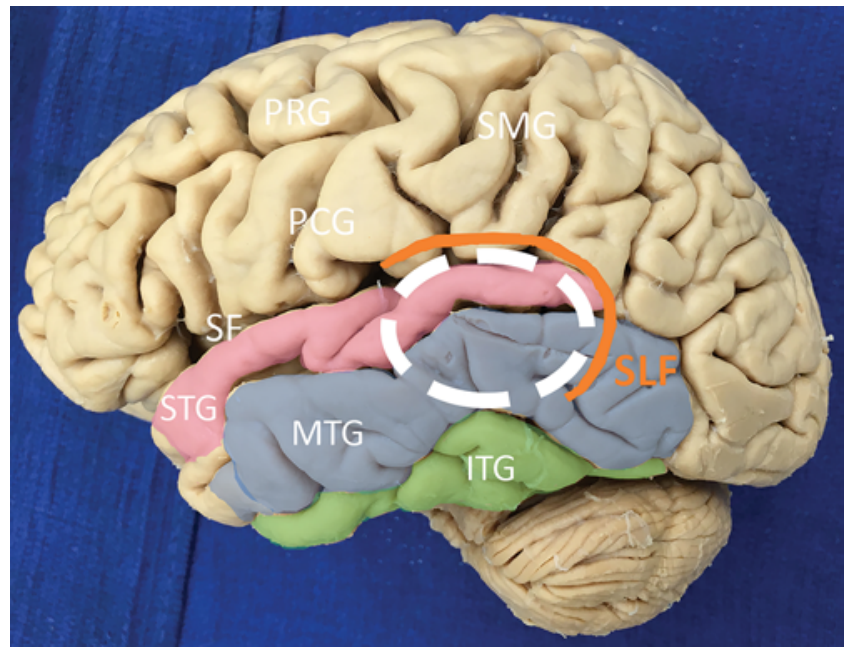

FIG. 2. Positioning of craniotomy relative to surrounding structures. The white dashed circle represents bone flap relative to the planned surgical disconnections. The bone flap allowed access to the anterior portion of the STG. The inferior border of the craniotomy was at the middle portion of the middle temporal gyrus (MTG). Care was taken so that the craniotomy was anterior and inferior to the path of the SLF, represented by orange. ITG = inferior temporal gyrus; $P C G=$ postcentral gyrus; $P R G=$ precentral gyrus; $\mathrm{SF}=$ sylvian fissure; $S M G=$ supramarginal gyrus. Pink = STG; blue = MTG; green = ITG . was made at the bottom of the field with a round cutting ball. The craniotomy was completed with a craniotome and was approximately $3 \mathrm{~cm}$ in diameter, as shown in Fig. 3 . We prefer this method, because some craniotomies are only slightly larger than a perforator bur hole.

Of note, in the event that a patient had undergone a prior biopsy or resection at another facility with a large, more traditional bone flap, we planned a new keyhole craniotomy rather than use the existing one. This typically resulted in the craniotomy being located within the prior bone flap. For instance, if a large question mark incision was previously made in the patient's scalp, we would open the bottom of the question mark for the second craniotomy.

The resection was performed with the microscope to allow for light and visualization through the smaller opening. An intraoperative procedure is demonstrated in Video 1.

VIDEO 1. Clip demonstrating the steps of a keyhole temporal lobe resection. Copyright Michael Sughrue, University of Oklahoma Health Sciences Center. Published with permission. Click here to view.

The posterior and superior disconnections were made first, separating eloquent white matter tracts and cortex from tumor. The dissection continued medially into the
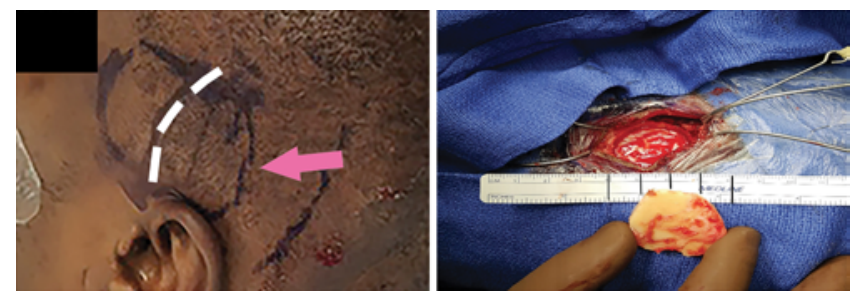

FIG. 3. Skin incision and craniotomy illustration. Left: Relative size of skin incision is shown with a white dashed line. Superficial mapping of the temporal lobe with a marker is designated with a pink arrow. Right: Example of craniotomy with bone flap. The bone flap is next to a ruler for comparison. Centimeter marks are illustrated on the ruler with black lines. The craniotomy measures roughly $3 \mathrm{~cm}$. 
TABLE 1. Patient characteristics

\begin{tabular}{|c|c|}
\hline Variable & No. $(\%)$ \\
\hline Total no. of patients & 52 \\
\hline Female & 14 \\
\hline Male & 38 \\
\hline \multicolumn{2}{|l|}{ Median age in yrs, range } \\
\hline All patients & $53,22-83$ \\
\hline Female & $45,33-77$ \\
\hline Male & $54,22-83$ \\
\hline \multicolumn{2}{|l|}{ Pathology } \\
\hline Glioblastoma & $35(67)$ \\
\hline Oligodendroglioma & $8(15)$ \\
\hline Astrocytoma & $9(17)$ \\
\hline \multicolumn{2}{|l|}{ WHO grade } \\
\hline IV & $35(67)$ \\
\hline II & $9(17)$ \\
\hline III & $8(15)$ \\
\hline \multicolumn{2}{|l|}{ Surgical history } \\
\hline No prior surgery & $26(50)$ \\
\hline Outside surgery & $26(50)$ \\
\hline \multicolumn{2}{|l|}{ Treatment prior to surgery } \\
\hline None & $33(63)$ \\
\hline Radiation & $3(6)$ \\
\hline Chemotherapy \& radiation & $13(25)$ \\
\hline Chemotherapy & $3(6)$ \\
\hline
\end{tabular}

temporal horn, as the tracts occurred laterally. As previously discussed, the hippocampus was used as a temporary landmark. In awake surgery, these cuts were monitored with subcortical speech, motor, and visuospatial mapping. The anterior temporal vein was coagulated and transected. Once this maneuver was completed, the anterior temporal lobe contents were reflected posteriorly and removed nearly en bloc. The hippocampus and amygdala could be resected using the subpial technique. However, this could be tailored based on surgeon preference. We chose to resect these structures even in the absence of tumor involvement based on studies that have reported reduced incidence of postoperative seizures with use of this practice. ${ }^{12}$ Notably, care was taken to avoid transgressing the pial border to prevent disturbance of the choroidal arteries and surrounding structures.

\section{Outcome Assessment}

Postoperatively, a physical therapist and speech language pathologist evaluated all patients. Preoperative task assessment (naming, motor function, and so on) was compared with postoperative task assessment. Patients underwent a full neurological examination by the attending neurosurgeon immediately after surgery, and at follow-up in the clinic within 3 months of surgery. Thus, complications were noted at the postoperative examination and in the clinic. Any complication that was reported following surgery but that had resolved by clinical follow-up was recorded as temporary. Surgery duration was recorded from skin opening to skin closure. The length of hospital stay was defined as the day of operation to the day of discharge.

Tumor volumes were calculated using pre- and postoperative contrast-enhanced T1-weighted MRI by J.D.B. and A.K.C. Tumor volume was agreed upon by the 2 investigators. For nonenhancing tumors, T2-weighted images were used. ImageJ (National Institutes of Health) was used for tumor segmentation, as performed by others. ${ }^{27}$ The tumor was outlined on individual slices by freehand. The volume was calculated as the sum of the individual areas multiplied by slice thickness. Residual tumor was calculated in the same manner, tracing areas of residual contrast enhancement or areas of $\mathrm{T} 2$ hyperintensity present in postoperative imaging. Distances were standardized for each imaging study to account for possible variability between baseline image sizes. The extent of resection (EOR) was calculated as follows: (preoperative tumor volume - postoperative tumor volume)/preoperative tumor volume. The EOR was recorded within the appropriate percentage range.

\section{Results \\ Patient Population}

A total of 52 patients with WHO Grade II-IV gliomas were treated with temporal keyhole lobectomy by the senior author (M.E.S.) between 2012 and 2015. Characteristics of these patients are given in Table 1. Thirty-five of 52 patients $(67 \%)$ were treated for glioblastoma, and 17 of $52(33 \%)$ were treated for oligodendroglioma or astrocytoma. Twenty-six of 52 patients $(50 \%)$ had not undergone a previous operation. Three of 52 patients $(6 \%)$ underwent radiation alone prior to resection. Thirteen of 52 patients (25\%) underwent radiation and chemotherapy prior to surgery, and 3 of $52(6 \%)$ underwent chemotherapy alone prior to operation. Tumor and operative data are given in Table 2. All patients had tumors of the temporal lobe; 30 of 52 tumors $(58 \%)$ were left sided and 22 of $52(42 \%)$ were right sided. The median tumor volume was $41 \mathrm{~cm}^{3}$; the largest tumor resected had a volume of $154.5 \mathrm{~cm}^{3}$.

\section{Operative Results}

Thirty of 52 patients (58\%) underwent awake surgery. The median duration was 250 minutes for patients undergoing awake and 224 minutes for patients undergoing asleep resection $(\mathrm{p}=0.12)$. Pre- and postoperative images from an illustrative case are shown in Fig. 4. The median length of hospital stay was 4 days. Ten of 52 patients $(19 \%)$ were discharged on postoperative days (PODs) $1-2,28$ of $52(54 \%)$ were discharged on PODs 3-6, 11 of $52(21 \%)$ were discharged on PODs 7-14, and 3 of $52(6 \%)$ were discharged on PODs 15-30.

EORs are noted in Table 3. Ninety to 100 percent of the preoperative tumor volume was resected in 35 of 52 patients (67\%), $70 \%-89 \%$ of tumor volume was resected in 16 of 52 patients (31\%), and $<69 \%$ of tumor volume was resected in 1 patient of $52(2 \%)$. The median EOR was $95 \%$. Three patients who missed postoperative clinic appointments and could not be contacted for rescheduling were considered lost to follow-up (6\%). The median follow-up for all patients was 14 months.

Postoperative complications are also noted in Table 3. 
TABLE 2. Tumor characteristics and operative data

\begin{tabular}{|c|c|}
\hline Variable & Value \\
\hline \multicolumn{2}{|l|}{ Tumor location, no. (\%) } \\
\hline $\mathrm{Lt}$ & $30(58)$ \\
\hline Rt & $22(42)$ \\
\hline \multicolumn{2}{|l|}{ Tumor vol, $\mathrm{cm}^{3}$} \\
\hline Median & 41.0 \\
\hline Range & $1.7-154.5$ \\
\hline Standard deviation & 35.4 \\
\hline \multicolumn{2}{|c|}{ Mapping, no. of patients (\%) } \\
\hline Awake surgery & $30(58)$ \\
\hline Asleep surgery & $22(42)$ \\
\hline \multicolumn{2}{|l|}{ Surgery duration, mins } \\
\hline Median awake & 250 \\
\hline Median asleep & 224 \\
\hline$p$ value & 0.12 \\
\hline Range & $107-457$ \\
\hline \multicolumn{2}{|c|}{ LOS in days, no. of patients (\%) } \\
\hline Median & 4 \\
\hline $1-2$ & $10(19)$ \\
\hline $3-6$ & $28(54)$ \\
\hline $7-14$ & $11(21)$ \\
\hline$>14$ & $3(6)$ \\
\hline
\end{tabular}

LOS $=$ length of hospital stay.

Postoperative neurosurgical complications included 3 patients $(6 \%)$ with a resection cavity hematoma, 1 of whom was taken back to the operating room for hematoma evacuation following hypertension in the recovery unit. Three patients $(6 \%)$ experienced contralateral hemiparesis, of whom 2 (4\%) suffered permanent deficits. Three patients $(5 \%)$ experienced early anomia that had improved at 4-week and 3-month follow-up. Four patients (8\%) developed hydrocephalus, of whom $3(6 \%)$ required placement of a ventriculoperitoneal shunt. Additionally, 1 patient (2\%) had temporary encephalopathy and 2 patients $(4 \%)$ experienced new-onset seizure.

Postoperative medical complications included 5 patients $(10 \%)$ with deep venous thrombosis found on routine surveillance scan. No postoperative epidural hematomas, pulmonary embolisms, or infections occurred in this series of patients.

\section{Discussion}

In this study, we present the intermediate-term results of patients who underwent temporal keyhole lobectomies for glioma resection. This technique limits bone flap and incision size with the intent that this will reduce the impact of surgery. We report both operative results and patient outcomes using this procedure, demonstrating the feasibility of safely resecting temporal gliomas with a less invasive approach.

In this series, the median EOR was $95 \%$, and $>70 \%$ of the tumor was resected in all except 1 case. Instances of incomplete tumor resection were due to eloquent location
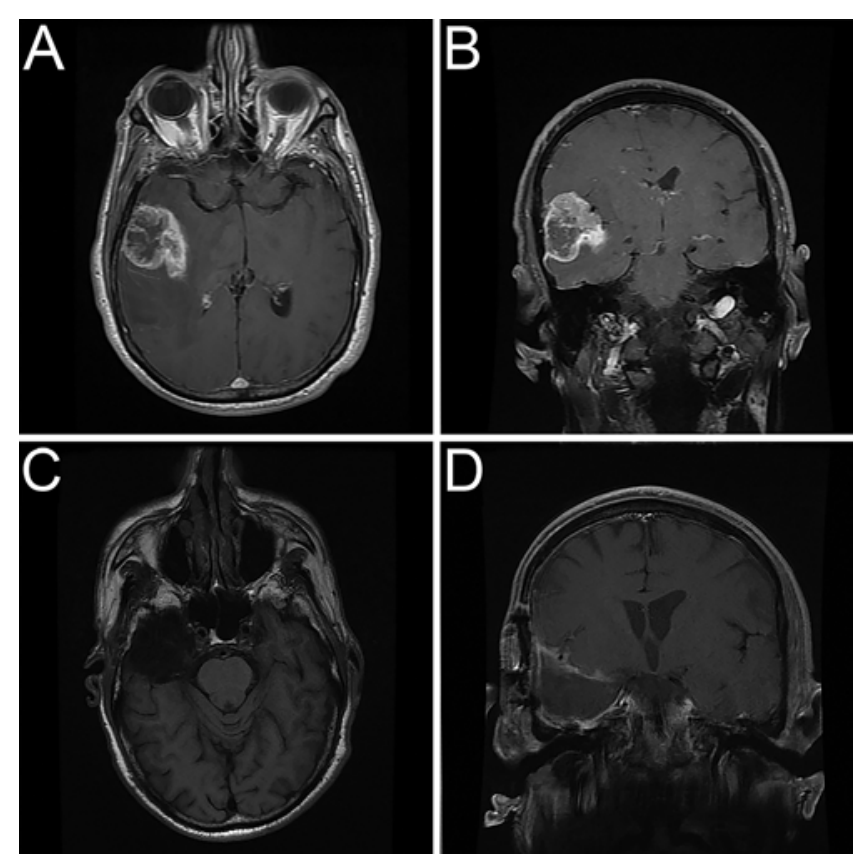

FIG. 4. Illustrative case of the temporal keyhole method used in a patient with a right temporal glioblastoma. Contrast-enhanced T1-weighted MR images show the axial $(\mathbf{A})$ and coronal $(\mathbf{B})$ views of a ring-enhancing lesion. T1-weighted FLAIR axial (C) and contrast-enhanced T1-weighted MR coronal (D) images illustrate postoperative resection.

TABLE 3. Surgical outcomes

\begin{tabular}{lc}
\hline \multicolumn{1}{c}{ Variable } & Value \\
\hline EOR, no. of patients (\%) ${ }^{*}$ & $35(67)$ \\
\hline $90 \%-100 \%$ & $16(31)$ \\
\hline $70 \%-89 \%$ & $1(2)$ \\
\hline$<70 \%$ & 14.0 \\
\hline Follow-up & $2-48$ \\
\hline Median, mos & 3 \\
\hline Range, mos & \\
\hline Did not follow-up, no. of patients & $3(6) / 0$ \\
\hline No. of patients w/ complications (\%), temporary/ \\
permanent $†$ \\
\hline Hemorrhage & $3(6) / 0$ \\
\hline Anomia & $1(2) / 0$ \\
\hline Encephalopathy & $2(4) / 0$ \\
\hline Seizure & $4(8) / 3(6)$ \\
\hline Hydrocephalus & $3(6) / 2(4)$ \\
\hline Weakness & $0(0) / 0$ \\
\hline Infection & $5(10) / 0$ \\
\hline Deep venous thrombosis
\end{tabular}

* The median EOR was $95 \%$.

† Some patients had $>1$ complication. Permanent complications included hydrocephalus requiring placement of a ventriculoperitoneal shunt and postoperative neurological deficits (weakness, aphasia, seizures, and so on) not resolved at 6-month follow-up. 
rather than inadequate exposure. Our data suggest that neither efficacy nor safety is compromised with the temporal keyhole method.

A multitude of intracranial pathologies requiring surgery can safely be addressed using a keyhole craniotomy., $7,8,10,11,16,18,21,24,26,28,36,38$ The term "keyhole" is relative because there is no size at which a craniotomy becomes too large or too small to be considered keyhole surgery. Rather, it is a philosophical approach to treating tumors, wherein the smallest feasible craniotomy is made to achieve the goals of resection. Regarding its use in temporal locations, keyhole principles have been applied to epilepsy surgery with a resultant decrease in operative duration and length of hospital stay. ${ }^{4,17,37}$

For our purposes, the keyhole temporal craniotomy dimensions, precise location, and resultant surgical trajectory are tailored to the patient's lesion and its relationship to surrounding white matter tracts as imaged by DTI. Due to the variability of these lesions, there is no standard temporal keyhole craniotomy that can be planned for all patients. Again, this supports the concept that keyhole neurosurgery is more of a philosophy that does not abide by strict surgical parameters. ${ }^{34}$ For instance, accomplishing maximum EOR in one patient may involve the craniotomy being placed slightly more posterior to expose the key area of tumor-eloquence disconnection near the SLF, as opposed to another patient whose SLF is more anteriorly based.

The mainstay of treatment for low- and high-grade gliomas is maximal resection because this has been repeatedly demonstrated to increase survival. ${ }^{1,13,15,29,30,35}$ Our median EOR of $95 \%$ is comparable to those of other studies, e.g., in which low-grade temporal gliomas had an EOR of 92.5\% with intraoperative MRI and $90.7 \%$ without. ${ }^{3}$ Neoplasm size did not preclude the use of this technique, because large tumors (up to $154.5 \mathrm{~cm}^{3}$ ) were safely resected with little residual. The median tumor volumes recorded in our study are comparable to those noted in previous studies. $3,15,33$

Careful preoperative planning and consideration of white matter tracts initially is critical in minimizing the size of the craniotomy while preserving function. The role of awake craniotomies using cortical and subcortical mapping with direct electrical stimulation is well established for tumors in eloquent areas. ${ }^{14}$ Our operative treatment paradigm uses both direct electrical stimulation and fiber tractography to define and avoid eloquent brain parenchyma. In our experience, fiber tractography accurately predicts the coordinates of eloquent regions. ${ }^{5}$ With these adjuncts, permanent deficits occurred in only 5 of 49 patients $(10 \%)$. We emphasize that these results can be achieved by careful planning aided by the use of intraoperative monitoring.

We did not observe any surgical site infections in this series, and our infection rate is favorable to those reported elsewhere..$^{2,9,22}$ It may be possible that keyhole techniques are associated with improved complication rates; however, our data set is not adequately powered to conclude this. Larger studies would be required to show significant reduction of complications.

Postoperative resection hematoma occurred in a small subgroup of patients, with 1 patient returning to the operating room for hematoma evacuation. One of the postoper-

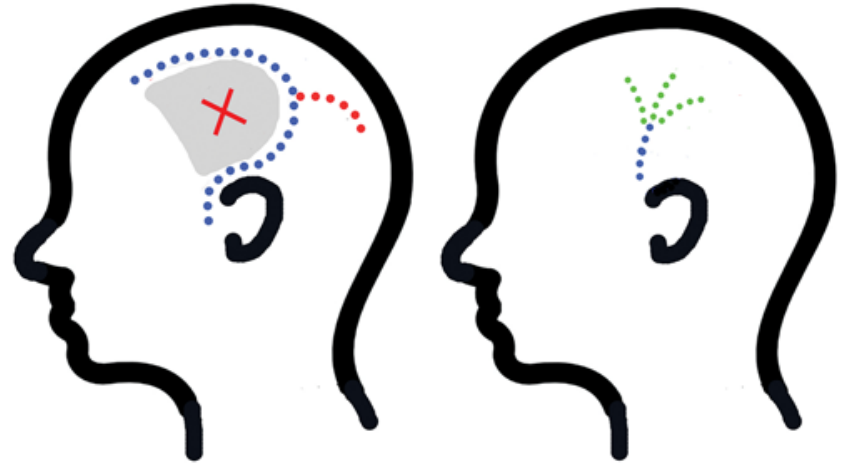

FIG. 5. Repeat craniotomy after initial resection. Left: Primary question mark incision (blue dotted line). A complex incision and craniotomy may limit subsequent craniotomies due to scarring of the dura and vascular issues related to wound healing. Repeat incisions within the gray area may heal poorly due to vascular supply. A repeat "T" incision (red dotted line) is possible but may also heal poorly. Right: Temporal keyhole lobectomy incision (blue dotted line). A smaller incision is less limiting on repeat craniotomies for recurrent tumors (green dotted lines).

ative resection hematomas occurred concurrently with the use of high-dose anticoagulation for deep venous thrombosis treatment in the early postoperative period. Since then, we have introduced stricter protocols in regard to postoperative anticoagulation.

Admittedly, it is difficult to quantitatively prove that a smaller craniotomy is more desirable than a traditional craniotomy. This is in part due to traditional craniotomies having acceptable outcomes. In this study, we focused on the equipoise of the technique, although we do believe that there are potential benefits of this operation when compared with the traditional approach. The benefits include less temporalis muscle dissection, an incision that is less cosmetically deforming, and a technique that simplifies wound issues. A smaller bone flap and incisional site becomes relevant with multiple resections and/or with the use of adjuvant therapies such as bevacizumab, which can increase wound healing complications. Furthermore, if a tumor recurs in a neighboring region, we find that a smaller incision closes fewer doors to future operations when compared with a large horseshoe or question mark incision. In our experience, large complex incisions limit subsequent craniotomies due to scarring of the dura mater and vascular issues related to wound healing. A schematic of the feasibility of repeat craniotomies after different incisions is shown in Fig. 5. This may lower the threshold for offering an additional surgery in the event of recurrence.

We note that although this study reports favorable outcomes in resecting gliomas with a temporal keyhole lobectomy approach, we do not attempt to provide evidence that this method is superior to traditional craniotomy. Instead, we sought to assess the outcomes achieved using a keyhole approach. Our results suggest that the treatment of gliomas can be safely and effectively pursued using a keyhole lobectomy.

\section{Conclusions}

We present evidence that low- and high-grade gliomas can be maximally resected by implementing the temporal 
keyhole approach without undue risk to the patient. Neurosurgeons can achieve outcomes similar to those with larger, traditional craniotomies in patients presenting with temporal gliomas.

\section{References}

1. Aghi MK, Nahed BV, Sloan AE, Ryken TC, Kalkanis SN, Olson JJ: The role of surgery in the management of patients with diffuse low grade glioma: A systematic review and evidence-based clinical practice guideline. J Neurooncol 125:503-530, 2015

2. Ahmadi R, Campos B, Haux D, Rieke J, Beigel B, Unterberg A: Assessing perioperative complications associated with use of intraoperative magnetic resonance imaging during glioma surgery-a single centre experience with 516 cases. Br J Neurosurg 30:397-400, 2016

3. Bai SC, Xu BN, Wei SH, Geng JF, Wu DD, Yu XG, et al: Intraoperative high-field magnetic resonance imaging combined with functional neuronavigation in resection of lowgrade temporal lobe tumors. World J Surg Oncol 13:286, 2015

4. Boling W: Minimal access keyhole surgery for mesial temporal lobe epilepsy. J Clin Neurosci 17:1180-1184, 2010

5. Bonney PA, Conner AK, Boettcher LB, Pittman N, Sughrue M: A simplified method of accurate postprocessing of diffusion tensor imaging for use in brain tumor resection. Operative Neurosurg [epub ahead of print], 2015

6. Charras P, Herbet G, Deverdun J, de Champfleur NM, Duffau $\mathrm{H}$, Bartolomeo P, et al: Functional reorganization of the attentional networks in low-grade glioma patients: a longitudinal study. Cortex 63:27-41, 2015

7. Clark JC, Spetzler RF: Defining the limits of the occipital transtentorial keyhole approach. World Neurosurg 80:6263, 2013

8. Daming C, Yiwen S, Bin Z, Yajun X, Jia Y, Rui S, et al: Large vestibular schwannoma resection through the suboccipital retrosigmoid keyhole approach. J Craniofac Surg 25:463-468, 2014

9. Dickinson H, Carico C, Nuño M, Mukherjee D, Ortega A, Black KL, et al: Unplanned readmissions and survival following brain tumor surgery. J Neurosurg 122:61-68, 2015

10. Ditzel Filho LF, McLaughlin N, Bresson D, Solari D, Kassam AB, Kelly DF: Supraorbital eyebrow craniotomy for removal of intraaxial frontal brain tumors: a technical note. World Neurosurg 81:348-356, 2014

11. Fischer G, Stadie A, Reisch R, Hopf NJ, Fries G, BöcherSchwarz H, et al: The keyhole concept in aneurysm surgery: results of the past 20 years. Neurosurgery 68 (1 Suppl Operative): $45-51,2011$

12. Ghareeb F, Duffau H: Intractable epilepsy in paralimbic Word Health Organization Grade II gliomas: should the hippocampus be resected when not invaded by the tumor? $\mathbf{J}$ Neurosurg 116:1226-1234, 2012

13. Hervey-Jumper SL, Berger MS: Role of surgical resection in low- and high-grade gliomas. Curr Treat Options Neurol 16:284, 2014

14. Hervey-Jumper SL, Li J, Lau D, Molinaro AM, Perry DW, Meng L, et al: Awake craniotomy to maximize glioma resection: methods and technical nuances over a 27 -year period. J Neurosurg 123:325-339, 2015

15. Ius T, Isola M, Budai R, Pauletto G, Tomasino B, Fadiga L, et al: Low-grade glioma surgery in eloquent areas: volumetric analysis of extent of resection and its impact on overall survival. A single-institution experience in 190 patients: clinical article. J Neurosurg 117:1039-1052, 2012

16. Kang HJ, Lee YS, Suh SJ, Lee JH, Ryu KY, Kang DG: Comparative analysis of the mini-pterional and supraorbital keyhole craniotomies for unruptured aneurysms with numeric measurements of their geometric configurations. J Cerebrovasc Endovasc Neurosurg 15:5-12, 2013

17. Little AS, Smith KA, Kirlin K, Baxter LC, Chung S, Maganti $\mathrm{R}$, et al: Modifications to the subtemporal selective amygdalohippocampectomy using a minimal-access technique: seizure and neuropsychological outcomes. J Neurosurg 111:1263-1274, 2009

18. Ma Y, Lan Q: An anatomic study of the occipital transtentorial keyhole approach. World Neurosurg 80:183-189, 2013

19. Mandonnet E, Sarubbo S, Duffau H: Proposal of an optimized strategy for intraoperative testing of speech and language during awake mapping. Neurosurg Rev 40:29-35, 2017

20. Marcus HJ, Cundy TP, Hughes-Hallett A, Yang GZ, Darzi A, Nandi D: Endoscopic and keyhole endoscope-assisted neurosurgical approaches: a qualitative survey on technical challenges and technological solutions. Br J Neurosurg 28:606-610, 2014

21. Marcus HJ, Sarkar H, Mindermann T, Reisch R: Keyhole supracerebellar transtentorial transcollateral sulcus approach to the lateral ventricle. Neurosurgery 73 (Operative Neurosurg 2):onsE295-onsE301, 2013

22. Marcus LP, McCutcheon BA, Noorbakhsh A, Parina RP, Gonda DD, Chen C, et al: Incidence and predictors of 30-day readmission for patients discharged home after craniotomy for malignant supratentorial tumors in California (19952010). J Neurosurg 120:1201-1211, 2014

23. Martino J, Vergani F, Robles SG, Duffau H: New insights into the anatomic dissection of the temporal stem with special emphasis on the inferior fronto-occipital fasciculus: implications in surgical approach to left mesiotemporal and temporoinsular structures. Neurosurgery 66 (3 Suppl Operative):4-12, 2010

24. Maurer AJ, Bonney PA, Strickland AE, Safavi-Abbasi S, Sughrue ME: Brainstem cavernous malformations resected via miniature craniotomies: technique and approach selection. J Clin Neurosci 22:865-871, 2015

25. Mori K: Keyhole concept in cerebral aneurysm clipping and tumor removal by the supraciliary lateral supraorbital approach. Asian J Neurosurg 9:14-20, 2014

26. Moscovici S, Mizrahi CJ, Margolin E, Spektor S: Modified pterional craniotomy without "MacCarty keyhole". J Clin Neurosci 24:135-137, 2016

27. Oya S, Kim SH, Sade B, Lee JH: The natural history of intracranial meningiomas. J Neurosurg 114:1250-1256, 2011

28. Reisch R, Fischer G, Stadie A, Kockro R, Cesnulis E, Hopf $\mathrm{N}$ : The supraorbital endoscopic approach for aneurysms. World Neurosurg 82 (6 Suppl):S130-S137, 2014

29. Sanai N, Berger MS: Extent of resection influences outcomes for patients with gliomas. Rev Neurol (Paris) 167:648-654, 2011

30. Sanai N, Berger MS: Glioma extent of resection and its impact on patient outcome. Neurosurgery 62:753-764, 264266, 2008

31. Sanai N, Berger MS: Intraoperative stimulation techniques for functional pathway preservation and glioma resection. Neurosurg Focus 28(2):E1, 2010

32. Sanai N, Mirzadeh Z, Berger MS: Functional outcome after language mapping for glioma resection. $\mathbf{N}$ Engl J Med 358:18-27, 2008

33. Smith JS, Chang EF, Lamborn KR, Chang SM, Prados MD, Cha S, et al: Role of extent of resection in the long-term outcome of low-grade hemispheric gliomas. J Clin Oncol 26:1338-1345, 2008

34. Teo C, Sughrue ME: Principles and Practice of Keyhole Brain Surgery, ed 1. Stuttgart: Thieme Publishers, 2015

35. Watts C: Surgical management of high-grade glioma: a standard of care. CNS Oncol 1:181-192, 2012

36. Wilson DA, Duong H, Teo C, Kelly DF: The supraorbital 
endoscopic approach for tumors. World Neurosurg 82 (6 Suppl):S72-S80, 2014

37. Yang PF, Zhang HJ, Pei JS, Lin Q, Mei Z, Chen ZQ, et al: Keyhole epilepsy surgery: corticoamygdalohippocampectomy for mesial temporal sclerosis. Neurosurg Rev 39:99-108, 2016

38. Yu LH, Yao PS, Zheng SF, Kang DZ: Retractorless surgery for anterior circulation aneurysms via a pterional keyhole approach. World Neurosurg 84:1779-1784, 2015

\section{Disclosures}

The authors report no conflict of interest concerning the materials or methods used in this study or the findings specified in this paper.

\section{Author Contributions}

Conception and design: Sughrue, Conner, Burks, Glenn. Acquisition of data: Smitherman, Pryor, Glenn, Briggs, Bonney. Analysis and interpretation of data: Conner, Burks. Drafting the article: Conner, Burks, Smitherman, Bonney. Critically revising the article: Conner, Baker. Reviewed submitted version of manuscript: Sughrue, Baker. Approved the final version of the manuscript on behalf of all authors: Sughrue.

\section{Supplemental Information \\ Videos}

Video 1. https://vimeo.com/205395015.

\section{Correspondence}

Michael E. Sughrue, Department of Neurosurgery, University of Oklahoma Health Sciences Center, 1000 N Lincoln Blvd., Ste. 4000, Oklahoma City, OK 73104. email: michael-sughrue@ouhsc. edu. 